RESEARCH

\title{
RELATIONSHIP OF DAILY TIME SPENT OUTDOORS WITH SLEEP QUALITY AND EMOTIONAL WELL-BEING AMONG COMMUNITY-DWELLING OLDER ADULTS DURING COVID-19 RESTRICTIONS
}

Turkish Journal of Geriatrics
DOI: $10.31086 /$ tjgeri.2021.240

2021; 24(3): 424-432

- Kenan Eren ŞANSAL ${ }^{1}$

- Leyla ATAŞ BALCI ${ }^{2}$

- Fadime ÇINAR ${ }^{3}$

- Dilber KARAGÖZOĞLU ÇOŞKUNSU ${ }^{4}$

- Sezin Hatice TANRIÖVER ${ }^{5}$

- Mehmet Bengü ULUENGIN ${ }^{5}$

CORRESPONDANCE

${ }^{1}$ Kenan Eren ŞANSAL

Yaşar University, Interior Architecture and

Environmental Design, Izmir, Turkey

Phone: +905343659277

e-mail: erensansal@gmail.com

Received: Aug 08, 2021

Accepted: Sep 01, 2021

${ }^{1}$ Yaşar University, Interior Architecture and Environmental Design, İmir, Turkey

2 Bahçescehir University, Physiotherapy and Rehabilitation, Istanbul, Turkey

${ }^{3}$ Nişantaşı University, Nursing, İstanbul, Turkey

${ }^{4}$ Fenerbahçe University, Physiotherapy and Rehabilitation, İstanbul, Turkey

${ }^{5}$ Bahçeşehir University, Interior Architecture and Environmental Design, İstanbul, Turkey

\section{Abstract}

Introduction: Given the recent constraints imposed due to the ongoing COVID-19 pandemic and the importance of the daily light-dark cycle for entraining the human circadian system, it is possible to state that the elderly are likely to be at serious risk of experiencing sleep-related problems. Whilst reduced or limited time outdoors, and thus, exposure to diurnal bright light appear to be detrimental to sleep and emotional well-being in old age, further research is required to confirm this relationship.

Materials and Method: To build on earlier work, a two-week study was conducted in a sample of 79 community-dwelling older adults recruited by using snowball sampling method. Throughout the study, the participants were allowed to be outdoors from 10.00 a.m. to 1.00 p.m. on weekdays. Whilst the participants were performing their daily habitual activities, they were asked to keep an activity diary and report on their sleep quality and emotional wellbeing using two questionnaires.

Results: The results demonstrated that participants' daily time spent outdoors (49.13 minutes on average) positively correlated with their perceived sleep quality and emotional well-being at various — but significant — levels $(p<0.05)$. Moreover, significant negative correlations were identified between participants' sleep quality and experienced emotional disturbances $(p<0.001)$.

Conclusion: Based on the research findings, it is reasonable to conclude that spending momentary periods outdoors may have adverse consequences for the elderly. To maintain a healthy sleep-wake cycle and alleviate related emotional problems in old age, increasing outdoor time and diurnal bright light exposure should be encouraged.

Keywords: Aged; COVID-19; Emotions; Photoperiod; Sleep; Social Isolation. 


\section{INTRODUCTION}

In industrialised nations, urban adult populations spend a substantial portion (approximately 90\%) of their day in buildings and other enclosed spaces, as evidenced by research on human activity patterns $(1,2)$. Inevitably, this type of lifestyle has led many people to perform most of their daily activities in dimly and artificially lit environments. Studies on diurnal light exposure patterns have provided empirical evidence that the daily duration of exposure to outdoor light levels $>1,000 \mathrm{~lx}$ is about 90 minutes in young and middle-aged adults (3) and can be as short as 3 minutes in the institutionalised elderly (4). Given the COVID-19-related preventive measures, such as precautionary self-isolation and lockdown globally taken to mitigate the ongoing outbreak, it seems reasonable to expect that the elderly are likely more at risk of beginning to spend less time outdoors and being confined to indoor spaces that lack daylight. Thus, the question arises as to whether these recent changes are inflicting an extra burden on the systemic health and well-being of the elderly.

Sleep is a biological need; it plays a major role in maintaining good health, including immunologic health, and quality of life (5). Elderly individuals with sleep problems are more prone to depression, cardiovascular diseases, cerebrovascular diseases, and hypertension (6). In humans, sleep is regulated by the reciprocal interaction between the homeostatic sleep drive, which tracks the amount of prior sleep, and the circadian system, which synchronises bodily processes with the 24-hour solar day (7). Whilst light is the most important exogenous stimulus for entraining the circadian system (8), its potency dramatically decreases with age. It has been demonstrated that the steady decline in both crystalline lens transmittance and pupil area reduces the circadian photoreception of a 65-year-old adult to roughly one-third of the amount in a young adult aged 25 (9). As light exposure becomes more criti- cal to a healthy sleep-wake cycle, and thus, general well-being in old age, it is plausible to assume that the precautions against the COVID-19 pandemic and, unavoidably, reduced or limited diurnal light exposure outdoors may lead to serious sleep and sleep-related health problems in the elderly.

A critical review of the existing literature on this subject reveals that inconsistent or equivocal results have been reported thus far. On one hand, several studies provide empirical evidence to discredit the assumption made about the inadequacy of light exposure and its implications. For example, Noi and colleagues (4) observed that, despite the significant difference between the length of morning exposure to illuminances $>1,000 \mathrm{~lx}$ in summer and winter, the actigraphic sleep metrics, subjective sleep quality, and emotional well-being of a small group of nursing home residents did not vary considerably with the season. On the other hand, other studies indicate that spending time outdoors and being exposed to daylight are of benefit to the elderly (10-13). The issue with these latter studies is that the optimal duration or frequency of outdoor exposure cannot be determined. In a recent study, Corley and colleagues (10) demonstrated that using home gardens more often during lockdown, compared to the pre-lockdown period, was significantly associated with better sleep quality and mental health amongst a sample of community-dwelling elderly adults. Whilst this finding is in support of a previous study suggesting that increasing the daily duration of exposure to high illuminances is related to ameliorated sleep and depression in old age (11), it is not in complete accord with the results of two similar studies $(12,13)$. Unlike the others, these two studies, conducted in long-term care facilities, showed that being outdoors and exposed to daylight for only 30-40 minutes in the morning was potent enough to improve sleep quality and that longer or more frequent light exposure might not be required. 
Due to the lack of available evidence in the existing literature, it is still unsafe to reach any conclusions on the possible link between low daily doses of light and experiencing sleep-related problems. Before we can achieve a more detailed understanding of the influence of light on the sleep-wake cycle of older adults, there is a necessity for additional research studies to yield more conclusive results. Therefore, to build on earlier work, the present study was conducted by recruiting a sample of community-dwelling adults aged $\geq 65$ over a two-week period. This paper reports the results of our study to test the following hypotheses:

Hypothesis 1. Older individuals spend only brief periods outdoors.

Hypothesis 2. Limited time outdoors, and thus, less exposure to daylight have an inverse relationship with initiating and maintaining nocturnal sleep and, as a direct consequence, perceived sleep quality.

Hypothesis 3. Experiencing sleep difficulties is accompanied by emotional disturbance(s).

\section{MATERIALS AND METHOD}

\section{Participants}

To minimise contact with the elderly as much as possible during the COVID-19 pandemic, the researchers utilised the snowball sampling method; this yielded a total of 98 community-dwelling elders residing in ten different cities in Turkey. Among them, nine volunteers failed to meet the inclusion criteria. Additionally, ten volunteers had difficulties in following the study protocol and withdrew from the study. The remaining participants, whose descriptive characteristics are given in Table 1, were:

a) aged $\geq 65$;

b) literate;

c) cognitively intact. Approximately two weeks prior to the study, the cognitive functioning of the elderly volunteers was assessed by administering a Turkish version of the Standardised Mini-Mental State Examination (SMMSE-T). The SMMSE-T, translated and validated by Güngen and colleagues (14), is composed of various questions and tests to measure performance across different cognitive ability domains. A SMMSE-T score of 30 indicates no impairment; a score of $23 / 24$ is considered to be the cut-off for the diagnosis of mild dementia in the Turkish population. Volunteers who scored $<24$ were excluded to screen out individuals with dementia;

d) not concurrently using more than five prescribed medications or any medication for sleep and depression;

e) not in poor health, based on scores from a general health profile. For profiling purposes, all volunteers reported on their physical and mental health status by completing a Turkish version of the Short Form 36 (SF-36T). The SF-36T, reported to be valid and reliable by Pinar (15), is a 36-item, self-administered questionnaire assessing perceived changes in health and general health across eight domains. For each domain, raw item scores are converted, summed, and then transformed into a single score ranging from zero (the worst possible health state) to 100 (the best possible health state). Participants' SF-36T scores were comparable to the normative data of Demiral and colleagues (16) for Turkish adults aged $\geq 65$. The domains for which relatively low scores were obtained were social functioning (62.50 vs. 83.70) and role emotional (62.02 vs. 86.50$) ;$ and

f) thoroughly informed about the study. As per the ethical clearance granted by Yaşar University Ethics Committee, written informed consent was obtained from each participant.

\section{Sleep Quality}

A Turkish version of the Richards-Campbell Sleep Questionnaire (RCSQ-T), shown to be reliable and valid by Özlü and Özer (17), was also com- 
Table 1. Descriptive characteristics of the participants $(n=79)$

\begin{tabular}{|c|c|c|c|c|c|c|}
\hline \multicolumn{2}{|c|}{ Variable } & \multicolumn{2}{|c|}{$\mathbf{n}$} & \multicolumn{3}{|c|}{$\%$} \\
\hline \multirow{2}{*}{ Gender } & Female & \multicolumn{2}{|c|}{44} & \multicolumn{3}{|c|}{55.7} \\
\hline & Male & \multicolumn{2}{|c|}{35} & \multicolumn{3}{|c|}{44.3} \\
\hline \multirow{6}{*}{ Education Status } & Literate & \multicolumn{2}{|c|}{5} & \multicolumn{3}{|c|}{6.3} \\
\hline & Primary school & \multicolumn{2}{|c|}{22} & \multicolumn{3}{|c|}{27.8} \\
\hline & Secondary school & \multicolumn{2}{|c|}{4} & \multicolumn{3}{|c|}{5.1} \\
\hline & High school & \multicolumn{2}{|c|}{4} & \multicolumn{3}{|c|}{5.1} \\
\hline & Graduate & \multicolumn{2}{|c|}{32} & \multicolumn{3}{|c|}{40.5} \\
\hline & Postgraduate & \multicolumn{2}{|c|}{12} & \multicolumn{3}{|c|}{15.2} \\
\hline \multirow{2}{*}{ Chronic Illness } & Yes & \multicolumn{2}{|c|}{55} & \multicolumn{3}{|c|}{69.6} \\
\hline & No & \multicolumn{2}{|c|}{24} & \multicolumn{3}{|c|}{30.4} \\
\hline \multicolumn{2}{|l|}{ Variable } & Mean & SD & Median & Minimum & Maximum \\
\hline \multicolumn{2}{|l|}{ Age (years) } & 71.62 & 4.90 & 71 & 65 & 92 \\
\hline \multicolumn{2}{|c|}{ Body Mass Index $\left(\mathrm{kg} / \mathrm{m}^{2}\right)$} & 26.78 & 3.56 & 26.43 & 20.96 & 34.37 \\
\hline \multicolumn{2}{|l|}{ SMMT-T Score } & 27.76 & 1.96 & 28 & 24 & 30 \\
\hline \multirow{8}{*}{ SF-36T Score } & Physical functioning & 75.88 & 20.44 & 80 & 0 & 100 \\
\hline & Role physical & 57.59 & 42.24 & 75 & 0 & 100 \\
\hline & Bodily pain & 61.44 & 25.83 & 62 & 0 & 100 \\
\hline & General health & 61.81 & 20.33 & 62 & 0 & 100 \\
\hline & Vitality & 59.93 & 19.00 & 60 & 10 & 100 \\
\hline & Social functioning & 62.50 & 31.39 & 62.50 & 0 & 100 \\
\hline & Role emotional & 62.02 & 41.93 & 66.66 & 0 & 100 \\
\hline & Mental health & 67.69 & 16.68 & 72 & 24 & 100 \\
\hline
\end{tabular}

SMMT-T: Standardised Mini-Mental Test; SF-36T: Short Form 36; SD: Standard deviation

pleted daily by the participants to evaluate previous night's sleep. The RCSQ-T is a five-item, visual analogue scale measuring perceived sleep depth, latency, efficiency, and overall sleep quality. A total score, ranging from $0-100$, is obtained by calculating the mean score for all items. A high total score represents a high level of sleep satisfaction.

\section{Daily Activity}

To measure time spent outdoors during daylight hours, the participants kept a daily activity diary devised by the authors. The diary comprises a table in which all activities performed over a day are reported. In each row of the table, information on activity definition, time, duration, and place is required. 
Based on the contemporaneous sunrise and sunset hours at the activity location, the amount of outdoor time for all daytime activities was calculated and used to obtain the cumulative total for a single day or daily time spent outdoors (DTSO).

\section{Emotional Well-Being}

A Turkish version of the Depression Anxiety Stress Scale (DASS-42T), translated and demonstrated to be psychometrically sound by Uncu and colleagues (18), was used to assess participants' emotional well-being. The DASS-42T is a self-administered instrument that is composed of three subscales measuring the negative emotional states of depression, anxiety and stress over the last seven days. Each subscale consists of 14 items rated on a four-point scale of severity. Subscale scores, varying between $0-42$, are obtained by adding the scores for each subscale item. Scores greater than nine, seven, and 14 were reported to be indicative of depression, anxiety and stress, respectively.

\section{Procedure}

From 1-7 February 2021, the cognitive functioning and perceived health status of the consenting volunteers were evaluated by administering the SMMSE-T and SF-36T in person or online. Moreover, data on volunteers' sociodemographic characteristics, body weight, height, chronic illnesses, and medication use were also gathered in this period. Following the screening for participation, a bound folder containing the daily activity diary, RCSO-T, and DASS-42T was posted to each participant. All participants were thoroughly informed about how to use the folder during the week prior to the study.

The study was conducted between 15 February-1 March 2021. During this period, due to the precautions against the spread of COVID-19 and the related restrictions, adults aged $\geq 65$ were only allowed to be outdoors from 10.00 a.m. to 1.00 p.m. on weekdays. This two-week period was considered to be long enough to understand the possible effects of imposed restrictions without jeopardising participant adherence, and, thus, it was employed for data collection. Throughout the study, the participants updated the activity diary daily from 1528 February 2021 and filled in the RCSQ-T each morning between the second and last day of the study. On the eighth and last day, the DASS-42T was self-administered to gain an insight into participants' emotional well-being over the first and second weeks of the study. Following the completion of the study, participants either sent back the folders by post or scanned them to transfer their data electronically. Official permission was granted by the Republic of Turkey Ministry of Health for the study and its procedure (Application No: 2021-0125T16_04_06).

\section{Statistical Analysis}

The Kolmogorov-Smirnov test and normality plots were used to assess the normality of the data. Continuous variables were reported by using means, standard deviations, medians, minimum values, and maximum values. Categorical variables were presented by using absolute and relative frequencies. Spearman's correlation coefficients were calculated to determine the relationships between participants' self-reported data obtained over two weeks. According to Pallant's (19) definition, the strength of these relationships was considered to be small, medium, and large for the ranges of 0.10 $0.29,0.30-0.49$, and $0.50-1$, respectively. All statistical analyses were carried out by using the Statistical Package for the Social Sciences (version 22.0; SPSS Inc., Chicago, IL, USA). The level of significance was set at $p<0.05$.

\section{RESULTS}

Participants' cumulative DTSO, RCSQ-T, and DASS$42 T$ scores were calculated (see Table 2). It was evident from the results that the average DTSO was 
Table 2. Participants' DTSO, RCSQ-T scores and DASS-42T scores $(n=79)$

\begin{tabular}{|l|c|c|c|c|c|}
\hline Variable & Mean & SD & Median & Minimum & Maximum \\
\hline DTSO (minutes) & 49.13 & 36.08 & 42.85 & 0 & 171.43 \\
\hline RCSQ-T Score & 71.52 & 14.26 & 70 & 42.43 & 100 \\
\hline Depression Score & 12.74 & 9.93 & 10.5 & 0 & 40 \\
\hline Anxiety Score & 10.81 & 10.30 & 6.5 & 0 & 41.50 \\
\hline Stress Score & 15.15 & 9.42 & 14 & 0 & 40 \\
\hline
\end{tabular}

DTSO: Daily time spent outdoors; RCSQT: Richards-Campbell Sleep Questionnaire; DASS-42T: Depression Anxiety Stress Scale; SD: Standard deviation

only 49.13 minutes; this was in support of Hypothesis 1. Whilst the participants did not report experiencing major sleep disturbances based on their RCSQ-T scores, they were mildly depressed, moderately anxious, and mildly stressed, according to their scores on the DASS-42T.

To test Hypothesis 2, the correlation of participants' DTSO scores with their RCSQ-T scores was also computed over the overall study period. The results were in support of the second hypotheses (Table 3). There was a weak — but significant - positive correlation between their DTSO scores and perceived sleep quality $(p<0.05)$. In addition, it was further identified that participants' DTSO was found to inversely correlate with their depression, anxiety, and stress scores at various - yet significant - levels ( $p<0.05)$. Moreover, participants' RCSQ-T and DASS-42T scores significantly and negatively correlated with each other at moderate or high levels $(p<0.001)$. These results were consistent with Hypothesis 3.

Table 3. Correlations among participants' DTSO, RCSQ-T scores and DASS-42T scores ( $n=79)$

\begin{tabular}{|c|c|c|c|c|c|c|}
\hline \multicolumn{2}{|l|}{ Variable } & \multirow{2}{*}{$\begin{array}{r}\text { DTSO } \\
1.000\end{array}$} & \multirow{2}{*}{$\begin{array}{c}\text { RCSQ-T Score } \\
0.288\end{array}$} & \multirow{2}{*}{$\begin{array}{c}\begin{array}{c}\text { Depression } \\
\text { Score }\end{array} \\
-0.322\end{array}$} & \multirow{2}{*}{$\begin{array}{c}\text { Anxiety Score } \\
-0.370\end{array}$} & \multirow{2}{*}{$\begin{array}{c}\text { Stress Score } \\
-0.250\end{array}$} \\
\hline & $r$ & & & & & \\
\hline & $\mathrm{p}$ & - & 0.010 & 0.004 & 0.001 & 0.026 \\
\hline \multirow{2}{*}{ RCSQ-T Score } & $r$ & 0.288 & 1.000 & -0.527 & -0.475 & -0.427 \\
\hline & $p$ & 0.010 & - & $<0.001$ & $<0.001$ & $<0.001$ \\
\hline \multirow{2}{*}{ Depression Score } & $r$ & -0.322 & -0.527 & 1.000 & 0.856 & 0.853 \\
\hline & $\mathrm{p}$ & 0.004 & $<0.001$ & - & $<0.001$ & $<0.001$ \\
\hline \multirow{2}{*}{ Anxiety Score } & $r$ & -0.370 & -0.475 & 0.856 & 1.000 & 0.836 \\
\hline & $\mathrm{p}$ & 0.001 & $<0.001$ & $<0.001$ & - & $<0.001$ \\
\hline \multirow{2}{*}{ Stress Score } & $r$ & -0.250 & -0.427 & 0.853 & 0.836 & 1.000 \\
\hline & $\mathrm{p}$ & 0.026 & $<0.001$ & $<0.001$ & $<0.001$ & - \\
\hline
\end{tabular}

DTSO: Daily time spent outdoors; RCSQ-T: Richards-Campbell Sleep Questionnaire; DASS-42T: Depression Anxiety Stress Scale; r: Spearman's rho 


\section{DISCUSSION}

One of the aims of our study was to determine the time that the elderly participants spent outdoors to estimate the duration of their outdoor daylight exposure during the ongoing COVID-19 restrictions. It was shown that the average DTSO was approximately 49 minutes during daylight hours - in other words, on average, our sample spent only $3.4 \%$ of a day in outdoor light levels. This finding is consistent with those of other researchers; for example, Obayashi and colleagues (20) demonstrated that the duration of daytime light exposure to illuminances $>1,000 \mathrm{~lx}$ was 50.3 minutes per day in a large sample of home-dwelling older adults. Given the importance of light to entrain the circadian system in humans, it is possible to expect that this limited exposure can induce or aggravate sleep disturbances and produce sleep-related emotional problems in the elderly.

The question of whether there is a link between daily light exposure and sleep-related problems in the elderly remains unresolved. However, our results indicate that there may be a significant association between these variables, which is well worth considering. Even though our sample reported fewer difficulties with sleep initiation and maintenance than their peers living independently and in long-term care facilities (21), the participants' DTSO significantly correlated with their sleep quality and their emotional state. Moreover, there were significant negative correlations between the RCSQ-T and DASS-42T scores. These results confirm the findings of other studies $(10,11)$, which suggest that increased time spent outdoors and bright natural light exposure is beneficial to nocturnal sleep, and thus, emotional well-being in community-dwelling elders.

Although it is possible to state that a brief exposure period $<1$ hour can be potent enough to elicit a significant circadian response based on our results and those of other studies $(12,13)$, it is too soon to reach a conclusion about the optimal duration of DTSO. Despite the relationship of participants' DTSO with their sleep quality and DASS-42 scores, it is not possible to state with certainty whether increasing the length of exposure will yield much better results due to the correlational nature of this study. In this regard, the literature provides unclear evidence to confirm or refute this assertion. Whilst it has been demonstrated that there may be an upper efficacious limit for daylight exposure (12), it has also been suggested that longer exposure periods are likely to be more effective $(11,22)$.

A major strength or contribution of this study is that it addresses an important gap in our knowledge by gathering data from elderly adults living in different regions of Turkey during the restrictions imposed on adults aged $\geq 65$ due to COVID-19 pandemic. Moreover, it assesses not only sleep but also emotional well-being of the participants. In addition to these strengths, it should also be noted here that, in interpreting the above-mentioned results, two inherent weaknesses, or at least limitations, warrant consideration. Firstly, because of the ongoing pandemic and its impact on participant recruitment, the study population did not truly represent the entire age spectrum of elderly adults. Only two participants were older than 80 years at the time of data collection. Therefore, it is unclear to what extent our results are valid for those in extreme old age. Secondly, approximately $70 \%$ of the sample had chronic diseases associated with old age and were on medication for these diseases. Whilst the participants were not using any antidepressants or sleep aids, they were taking medications such as $\beta$-blockers, which interfere with nocturnal sleep (23). Apart from its weaknesses or limitations, it is important to note here that employing actigraphy can also be considered for objectively delineating both sleep and light exposure patterns (24) in future studies and comparing their results with our RCSQ-T and DTSO results.

Even though further interdisciplinary research is required to fully apprehend the effects of time 
spent outdoors on the older population, one can safely draw two main conclusions from the results discussed above. Firstly, it is reasonable to conclude that spending only brief periods outdoors is likely to be detrimental to sleep and emotional well-being in the elderly. Before it was feasible to use electric lighting to compensate for the lack of daylight in buildings and enable living indoors, the sun had been our primary source of light. Therefore, from an evolutionary perspective, older adults should be encouraged to spend more time outdoors to reduce the extra burden on their health imposed by the

\section{REFERENCES}

1. Matz CJ, Stieb DM, Davis K et al. Effects of age, season, gender and urban-rural status on time-activity: Canadian Human Activity Pattern Survey (CHAPS 2). Int J Environ Res Public Health 2014;11(2):2108-2124. (PMID:24557523).

2. Schweizer C, Edwards RD, Bayer-Oglesby L et al. Indoor time-microenvironment-activity patterns in seven regions of Europe. J Expo Sci Environ Epidemiol 2007;17(2):170-181. (PMID:16721413)

3. Kawinska A, Dumont M, Selmaoui B, Paquet J, Carrier J. Are modifications of melatonin circadian rhythm in the middle years of life related to habitual patterns of light exposure? J Biol Rhythms 2005;20(5):451460. (PMID:16267384).

4. Noi A, Roe J, Gow A, McNair D, Aspinall P. Seasonal differences in light exposure and associations with health and well-being in older adults: An exploratory study. HERD 2017;10(5):64-79. (PMID:29056090).

5. Grandner MA. Sleep, health and society. Sleep Med Clin 2017;12(1):1-22. (PMID:28159089).

6. Bloom HG, Ahmed I, Alessi CA et al. Evidence-based recommendations for the assessment and management of sleep disorders in older persons. J Am Geriatr Soc 2009;57(5):761-789. (PMID:19484833).

7. Deboer T. Sleep homeostasis and circadian clock: Do the circadian pacemaker and the sleep homeostat influence each other's functioning? Neurobiol Sleep Circadian Rhythms 2018;5:68-77. (PMID:31236513).

8. Blume C, Garbazza C, Spitschan M. Effects of light on human circadian rhythms, sleep and mood. Somnologie 2019;23(3):147-156. (PMID:31534436).
COVID-19 pandemic and the related precautions (i.e. social isolation). Secondly, given that spending time outdoors and being exposed to bright diurnal light may greatly benefit the elderly, we can be fairly certain that the successful incorporation of daylight into the built-environment is crucial for older adults, especially for those with mobility impairments.

\section{Conflict of Interest}

The authors declare no conflicts of interest.

9. Turner PL, Mainster MA. Circadian photoreception: Ageing and the eye's important role in systemic health. $\mathrm{Br} J$ Ophthalmol 2008;92(11)1439-1444. (PMID:18757473).

10. Corley J, Okely JA, Taylor AM et al. Home garden use during COVID-19: Associations with physical and mental wellbeing in older adults. Journal of Environmental Psychology 2021;73:1-8. (DOI:10.1016/j. jenvp.2020.101545).

11. Wallace-Guy GM, Kripke DF, Jean-Louis G, Langer RD, Elliott JA, Tuunainen A. Evening light exposure: Implications for sleep and depression. J Am Geriatr Soc 2002;50(4):738-739. (PMID:11982677).

12. Düzgün G, Durmaz Akyol S. Effects of natural sunlight on sleep problems and sleep quality of the elderly staying in the nursing home. Holist Nurs Pract 2017;31(5):295-302. (PMID:28786887).

13. Şansal KE, Tanriöver SH, Aka BT. Effects of outdoor natural light exposure on sleep quality in the elderly. Turkish Journal of Geriatrics 2020;23(1):138-146. (DOI:10.31086/tjgeri.2020.147).

14. Güngen C, Ertan T, Eker E, Yaşar R, Engin F. Reliability and validity of the Standardized Mini Mental State Examination in the diagnosis of mild dementia in Turkish population. Turk Psikiatri Derg 2002;13(4):273-281. (PMID:12794644).

15. Pinar R. Reliability and construct validity of the SF-36 in Turkish cancer patients. Qual Life Res 2005;14(1):259-264. (PMID:15789959).

16. Demiral Y, Ergor G, Unal B, et al. Normative data and discriminative properties of Short Form 36 (SF36) in Turkish urban population. BMC Public Health 
2006;6:247. (PMID:17029646).

17. Özlü ZK, Özer N. Richard-Campbell Sleep Questionnaire validity and reliability study. J Turk Sleep Med 2015;2(2):29-32. (DOl:10.4274/jtsm.02.008).

18. Uncu $Y$, Bayram N, Bilgel N. Job related affective well-being among primary health care physicians. Eur J Public Health 2007;17(5):514-519. (PMID:17185328).

19. J Pallant. SPSS Survival Manual: A step by step guide to data analysis using SPSS for Windows. 3th edition, Open University Press, England 2007.

20. Obayashi K, Saeki K, Iwamoto J, Ikada Y, Kurumatani $\mathrm{N}$. Exposure to light at night and risk of depression in the elderly. J Affect Disord 2013;151(1):331-336. (PMID:23856285).
21. Haesler EJ. Effectiveness of strategies to manage sleep in residents of age care facilities. JBI Libr Syst Rev 2004;2(4):115-183. (PMID:27820000).

22. Hood B, Bruck D, Kennedy G. Determinants of sleep quality in the healthy aged: The role of physical, psychological, circadian and naturalistic light variables. Age Ageing 2004;33(2):159-165. (PMID:14960432).

23. Roepke SK, Ancoli-Israel S. Sleep disorders in the elderly. Indian J Med Res 2010;131:302-310. (PMID:20308755).

24. Ancoli-Israel S, Cole R, Alessi C, Chambers M, Moorcroft W, Pollak CP. The role of actigraphy in the study of sleep and circadian rhythms. Sleep 2003;26(3):342392. (PMID: 12749557). 